\title{
Fermions and Bosons in a Double-Well Potential
}

\author{
A. Bielas AND M.M. MAŚKA* \\ Department of Theoretical Physics, Institute of Physics, University of Silesia, \\ Uniwersytecka 4, 40-007 Katowice, Poland
}

The boson-fermion model, describing a mixture of fermions and bosons is analyzed on a small, two-site lattice. The model includes all the on-site Coulomb-type interactions between bosons, between fermions and between fermions and bosons. Additionally, the Hamiltonian includes a term that describes a conversion of a pair of opposite-spin fermions into a boson and vice versa. We show how this conversion depends on the model parameters.

DOI: 10.12693/APhysPolA.126.A-118

PACS: 67.85.-d, 67.85.Pq, 73.20.Jc

\section{Introduction}

A simple quantum mechanical problem of a particle in a double-well potential becomes a non-trivial challenge when the number of particles increases and a mixture of fermions and bosons is allowed [1]. The problem can be further complicated by taking into account the possibility that a pair of fermions is converted into a boson and vice versa [2]. Next, such dimers can be coupled to form an array of double-well potentials, which would lead to an extremely rich behavior.

The recent advances in laser technology allow for experimental realization of not only a single double-well potential, but an array of coupled double-wells [3]. One-, two- and three-dimensional double-well optical lattices can be constructed [4-9]. When tunneling between the local double-well potentials is negligible compared to tunneling inside the double-well potential, the system can be described as an assemble of individual independent two-site lattices (dimers). Apart from the possibility of an experimental realization in optical lattice, such a small system usually does not constitute a very realistic system; however, dimer calculations have an illustrious history for providing insight into models [10-12].

The conversion of fermionic pairs into bosons has been postulated in the "negative- $U$ " scenario of the high- $T_{\mathrm{c}}$ superconductivity [2]. More recently, it has been demonstrated that the Feshbach resonance $[13,14]$ and the stimulated Raman adiabatic passage process [15-17] can be used to produce bosonic diatomic molecules in the ground state by tightly binding fermionic atoms. The efficiency of the molecule production was proven to be strongly dependent on the interactions present in the system $[18,19]$. Therefore it is important to take all of them into account while studying the properties of the Fermi-Bose mixtures.

In this paper we address the problem of mixtures of interacting fermions and bosons in a double-well potential. We start with a system described by the Fermi-Bose-

${ }^{*}$ corresponding author; e-mail: maciek@phys.us.edu.pl
Hubbard model on a single dimer. Next, we add to the Hamiltonian the term responsible for an exchange of a pair of opposite-spin fermions into a hard-core and soft-core boson. We study the ground state as a function of the model parameters. In particular, we are interested in the process of the fermion-boson exchange.

\section{The fermion-boson model on a dimer}

We start our analysis from a fermion-boson model in a double-well potential. The system is described by the following Hamiltonian:

$$
\begin{aligned}
H & =-J_{\mathrm{f}} \sum_{\sigma=\uparrow, \downarrow}\left(c_{1 \sigma}^{\dagger} c_{2 \sigma}+c_{2 \sigma}^{\dagger} c_{1 \sigma}\right)+U_{\mathrm{ff}} \sum_{i=1,2} n_{i \uparrow}^{\mathrm{f}} n_{i \downarrow}^{\mathrm{f}} \\
& -J_{\mathrm{b}}\left(b_{1}^{\dagger} b_{2}+b_{2}^{\dagger} b_{1}\right)+\frac{U_{\mathrm{bb}}}{2} \sum_{i=1,2} n_{i}^{\mathrm{b}}\left(n_{i}^{\mathrm{b}}-1\right) \\
& +U_{\mathrm{bf}} \sum_{i=1,2} n_{i}^{\mathrm{f}} n_{i}^{\mathrm{b}}+g \sum_{i=1,2}\left(b_{i}^{\dagger} c_{i \uparrow} c_{i \downarrow}+c_{i \downarrow}^{\dagger} c_{i \uparrow}^{\dagger} b_{i}\right) .
\end{aligned}
$$

Here, $c_{i \sigma}^{\dagger}\left(c_{i \sigma}\right)$ creates (annihilates) a spin- $\sigma$ fermion at lattice site $i(i=1,2), b_{i}^{\dagger}\left(b_{i}\right)$ creates (annihilates) a boson at lattice site $i, J_{\mathrm{f}}$ and $J_{\mathrm{b}}$ represent the hopping integrals for fermions and bosons, respectively. In the following we chose $J_{\mathrm{f}}=J_{\mathrm{b}}$ to be the energy unit. All the local interactions are taken into account: $U_{\mathrm{ff}}, U_{\mathrm{bb}}$ and $U_{\mathrm{bf}}$ are on-site fermion-fermion, boson-boson, and boson-fermion interactions, respectively. The first line in Eq. (1) describes fermions, the second one bosons and the third one the boson-fermion interaction. This interspecies interaction includes the Coulomb interaction and exchange processes converting pairs of itinerant oppositespin fermions into hard-core bosons and vice versa. The coupling parameter $g$ describes the magnitude of this process. The number of fermions is limited to four by the Pauli exclusion principle, but we allow up to 50 bosons. If there is no exchange between fermions and bosons $(g=0)$ the number of fermions $N^{\mathrm{f}}$ and number of bosons $N^{\mathrm{b}}$ are conserved separately. When $g \neq 0, N^{\mathrm{f}}+2 N^{\mathrm{b}}$ is conserved. The Hamiltonian is numerically diagonalized in the space spanned by vectors $|i\rangle^{\mathrm{f}} \otimes|j\rangle^{\mathrm{b}}$, where $|i\rangle^{\mathrm{f}} \equiv\left|n_{1 \uparrow}^{\mathrm{f}}, n_{1 \downarrow}^{\mathrm{f}}, n_{2 \uparrow}^{\mathrm{f}}, n_{2 \downarrow}^{\mathrm{f}}\right\rangle\left(n_{i \sigma}^{\mathrm{f}}=0,1\right)$ describes the state of the fermionic subsystem, whereas $|j\rangle^{\mathrm{b}}=\left|n_{1}^{\mathrm{b}}, n_{2}^{\mathrm{b}}\right\rangle$ are the boson states with $n_{1}^{\mathrm{b}}=0, \ldots, N^{\mathrm{b}}, n_{2}^{\mathrm{b}}=N^{\mathrm{b}}-n_{1}^{\mathrm{b}}$. 
The states of the entire system are described by the coefficients $\alpha_{i j}$ :

$$
|\Psi\rangle=\sum_{i j} \alpha_{i j}|i\rangle^{\mathrm{f}} \otimes|j\rangle^{\mathrm{b}} .
$$

\subsection{The case of $g=0$}

We start our study in a limit where pairs of fermions cannot be converted into bosons $(g=0)$. The system has been diagonalized in subspaces with $0,1,2,3$ and 4 fermions and 4 bosons. In the absence of fermions the ground state is entirely defined by the strength of the inter-boson interaction $U_{\mathrm{bb}}$. For $U_{\mathrm{bb}}>0$ in the ground state there are $N^{\mathrm{f}} / 2$ bosons at the left site and the same number at the right site. In the limit of strong inter-boson repulsion we have

$$
\left|\Psi_{\mathrm{GS}}\right\rangle=\left|N^{\mathrm{b}} / 2, N^{\mathrm{b}} / 2\right\rangle \text {. }
$$

However, if the interaction becomes sufficiently negative $\left(U_{\mathrm{bb}} \lesssim-1\right)$, the ground state evolves into the Schrödinger cat state in the form of a superposition of states with all bosons at the left site and all bosons at the right site. In the limit of a strong attraction the ground state is given by

$$
\left|\Psi_{\mathrm{GS}}\right\rangle=\frac{1}{\sqrt{2}}\left(\left|N^{\mathrm{b}}, 0\right\rangle+\left|0, N^{\mathrm{b}}\right\rangle\right) .
$$

The evolution of the ground state as a function of $U_{\mathrm{bb}}$ is shown in Fig. 1. This plot shows coefficients $\left|\alpha_{j}\right|^{2}$ defined by

$$
|\Psi\rangle=\sum_{j} \alpha_{j}\left|N^{\mathrm{b}}-j, j\right\rangle .
$$

Since in this case we have no fermions, as opposed to the general form given by Eq. (2), only one index enumerates the basis vectors.

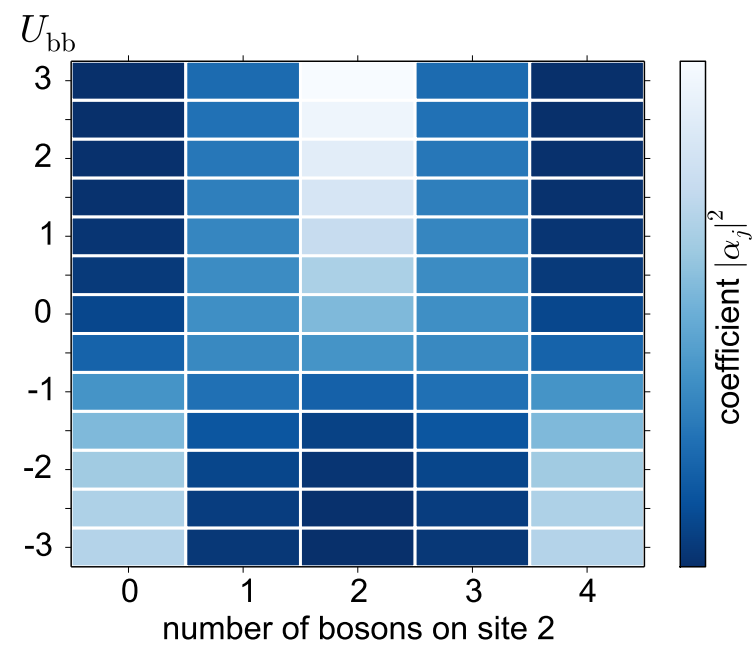

Fig. 1. The ground state of a system composed of 4 interacting bosons as a function of the strength of the interaction $U_{\mathrm{bb}}$.

Next, we introduce fermions to the system. The ground states for all allowed numbers of fermions are presented in Fig. 2. Here, $\left|\alpha_{i j}\right|^{2}$ are presented for $U_{\mathrm{ff}}=2$, $U_{\mathrm{bb}}=0.1$ and $U_{\mathrm{bf}}=4$. In the case of spin degeneracy only one of the equivalent states is presented.

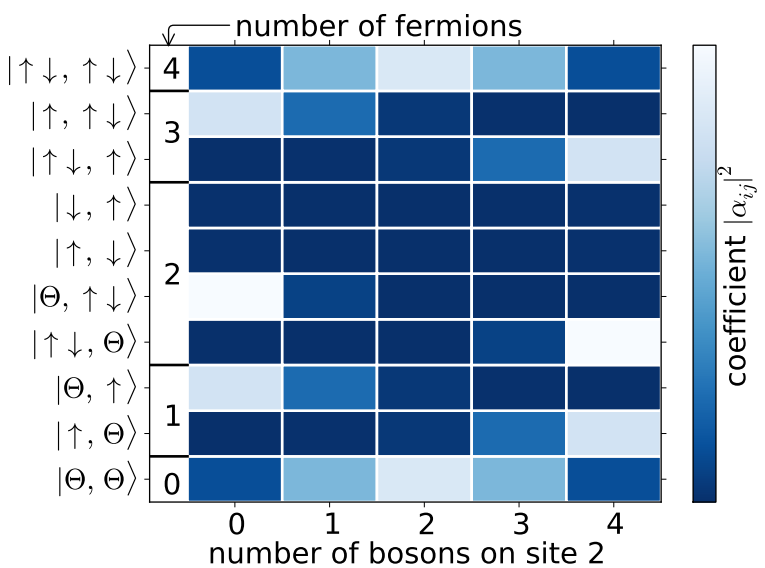

Fig. 2. The ground state of a system with 0 to 4 fermions and 4 bosons for $U_{\mathrm{ff}}=2, U_{\mathrm{bb}}=0.1, U_{\mathrm{bf}}=4$.

In this figure one can notice the obvious symmetry between the states with $N^{\mathrm{f}}$ and $4-N^{\mathrm{f}}$ fermions. What is interesting is the difference between the states with $N^{\mathrm{f}}=0$ or 4 and states with $N^{\mathrm{f}}=1,2$ or 3 . The inter-boson interaction $U_{\mathrm{bb}}=0.1$ is too positive to produce the Schrödinger's cat state $[20,21]$ in the absence of fermions. The same situation is for 4 fermions, when the only configuration allowed by the Pauli principle consists of two fermions sitting at site 1 and the other two at site

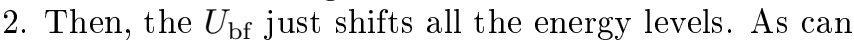
be seen in the lowest and highest rows in Fig. 2, in this case the ground state is peaked at $n_{1}^{\mathrm{b}}=n_{2}^{\mathrm{b}}=2$.

On the other hand, the presence of 1,2 or 3 fermions leads to the cat-like states, where the ground state is a superposition of a state with all bosons mostly at site 1 and fermion(s) at site 2 and a state with all bosons mostly at site 2 and fermion(s) at site 1 . This is especially visible for $N^{\mathrm{f}}=2$, when the main contribution to the ground state comes from an equal superposition of clearly distinguishable components

$$
\begin{aligned}
& \left|\Psi_{\mathrm{GS}}\right\rangle= \\
& \quad \frac{1}{\sqrt{2}}\left(|\Theta, \uparrow \downarrow\rangle^{\mathrm{f}} \otimes|4,0\rangle^{\mathrm{b}}+|\uparrow \downarrow, \Theta\rangle^{\mathrm{f}} \otimes|0,4\rangle^{\mathrm{b}}\right) .
\end{aligned}
$$

Such a configuration in a bulk system would correspond to a superposition of two equivalent chessboard states, where fermions occupy one sublattice and bosons the other one $[22,23]$.

A similar behavior can be observed for a larger number of bosons. Figure 3 shows the ground state for $0 \div 4$ fermions and 50 bosons. Also in this case the cat-like nature of the ground state is most pronounced for $N^{\mathrm{f}}=2$. Due to the number of bosons the boson-fermion repulsion dominates over the inter-fermion repulsion, which leads to ground-state configurations with both fermions sitting at the same site. 


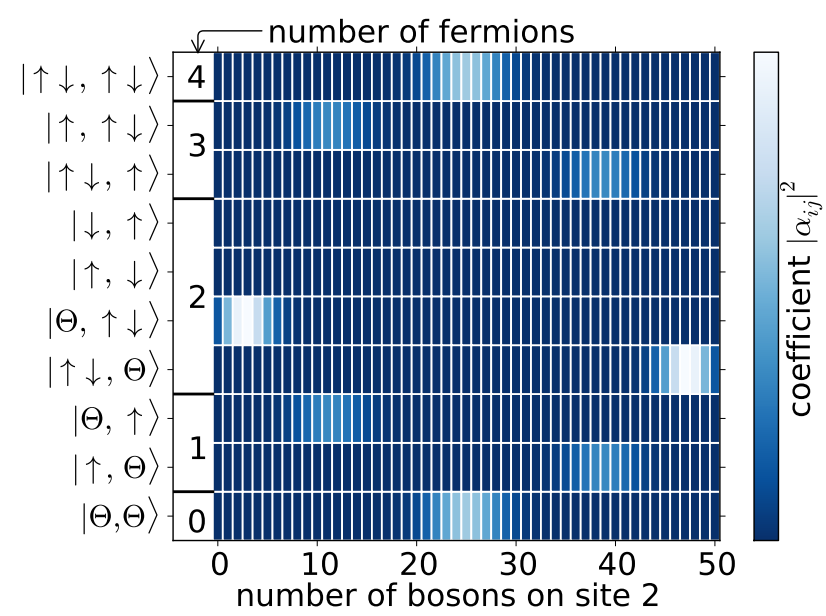

Fig. 3. The ground state of a system with 0 to 4 fermions and 50 bosons for $U_{\mathrm{ff}}=5, U_{\mathrm{bb}}=0.1, U_{\mathrm{bf}}=4$.

\subsection{The case of $g \neq 0$}

For $g \neq 0$ a pair of opposite-spin fermions can be changed into a boson and vice versa. Since the bosons represent pairs of fermions, we assume them to be hard-core bosons. Due to the exchange process the Hamiltonian does not conserve the numbers of fermions and bosons separately, only $2 N^{\mathrm{b}}+N^{\mathrm{f}}$ is conserved. We start the study of the fermions-boson conversion with the case of $2 N^{\mathrm{b}}+N^{\mathrm{f}}=2$. For a two-site lattice only in this case the fermion-fermion interaction may lead to a non-trivial effects in the absence of bosons: for $N^{\mathrm{f}}=1$ there is no fermion-fermion interaction, for $N^{\mathrm{f}}=3$ one of the lattice sites is singly occupied by a fermion, whereas the other site is doubly occupied. It leads to a constant interaction energy, independent of the configuration of fermions. Also, if 2 out of the 3 fermions are converted into a boson, the remaining fermion does not have a partner to interact with. For $N^{\mathrm{f}}=4$ both the sites are doubly occupied and a "band insulator" is formed. This would be also the case if all the four bosons are changed into a pair of hard-core bosons. However, one should note that for $2 N^{\mathrm{b}}+N^{\mathrm{f}}=2$ the boson-fermion and boson-boson interactions are unimportant. The boson-boson interaction $U_{\mathrm{bb}}$ is excluded by the assumption of the hard-core nature of the bosons. In order to study the influence of $U_{\mathrm{bf}}$ on the fermion-boson exchange one should take into account the case of $2 N^{\mathrm{b}}+N^{\mathrm{f}}=3$.

Figure 4 shows the number of fermions and bosons as a function of $U_{\mathrm{ff}}$ under the constraint $2 N^{\mathrm{b}}+N^{\mathrm{f}}=2$. It can be seen there that increasing inter-fermion repulsion drives the system from fermion-dominated towards boson-dominated. The states that contribute to the ground state for different values of $U_{\mathrm{ff}}$ for $g=0.4$ are presented in Fig. 5.

Since the hopping integrals have been assumed to be equal for bosons and fermions, in the absence of any interaction it is energetically favorable to have two fermions

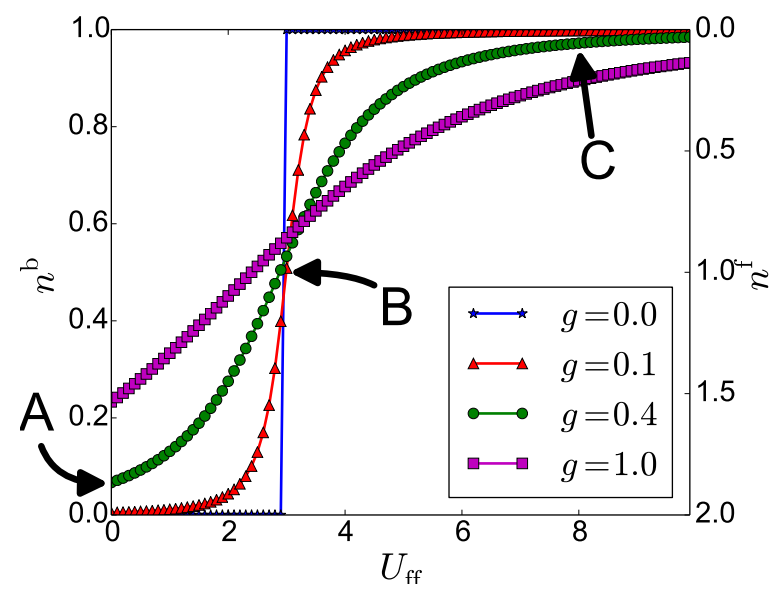

Fig. 4. The number of hard-core bosons $n_{\mathrm{b}}$ and the number of fermions $n_{\mathrm{f}}$ in the ground state as functions of the inter-fermion interaction $U_{\text {ff }}$ for different values of the fermion-boson coupling $g$. Letters A, B and C indicate points for which the decomposition of the ground state is presented in Fig. 5.

than a single boson in the system. This situation is shown in Fig. 5A. But then, the increasing repulsion starts to suppress the fermion mobility, while the boson mobility remains unaffected. As a result the fermions are gradually converted into a boson. Figure 5B shows an example of such a situation. Finally, when the interaction is so strong that the fermions are almost completely localized (which corresponds to the Mott insulator state in a half-filled bulk system), we have only a boson in the ground state - see Fig. 5C. One should note that this is a cat-state similar to the state described by Eq. (4). Here, however, there is no attractive inter-boson interaction.

In Fig. 4 we see that the fermion-boson conversion is strongly dependent on the coupling $g$. For small $g$ there is a sharp change of the boson and fermion occupation numbers at the threshold value of $U_{\mathrm{ff}}$. This is a remnant of the $g=0$ case, where $N^{\mathrm{f}}$ and $N^{\mathrm{b}}$ are conserved independently. For larger $g$ the exchange process dominates and even for $U^{\mathrm{ff}}=0$ there is a significant boson contribution to the ground state. With increasing $U_{\text {ff }}$ this contribution smoothly increases, diminishing the fermionic component. Nevertheless, even in the regime of strong inter-fermion repulsion a substantial fermion density is generated by the exchange term.

The next case to study is $2 N^{\mathrm{b}}+N^{\mathrm{f}}=4$. For hardcore bosons we have two limits: $N^{\mathrm{f}}=4, N^{\mathrm{b}}=0$ and $N^{\mathrm{f}}=0, N^{\mathrm{b}}=2$ and both of them represent a band insulator. Therefore, in these limiting cases the kinetic energy is equal to zero. However, apart from them, a configuration with one boson and a pair of opposite-spin fermions is possible. In this case both kinds of particles can be itinerant and the inter-species on-site interaction $U_{\text {bf }}$ becomes meaningful. Fig. 6 shows the ground-state boson and fermion occupation numbers as functions of $U_{\text {ff }}$ for $U_{\mathrm{bf}}=7$. 

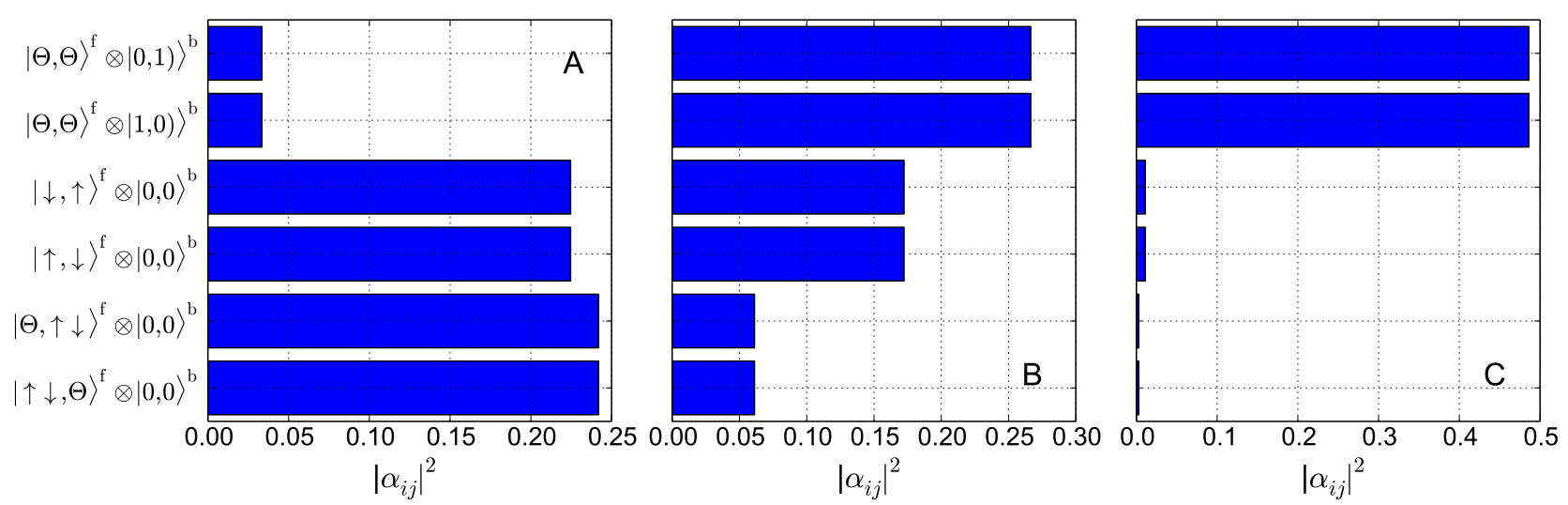

Fig. 5. The coefficients $\left|\alpha_{i j}\right|^{2}$ [Eq. (2)] of the ground state for $U_{\mathrm{ff}}=0$ (A), 3 (B), and 8 (C). These parts correspond to points marked by letters A, B and C in Fig. 4. In all cases $g=0.4$ is assumed.

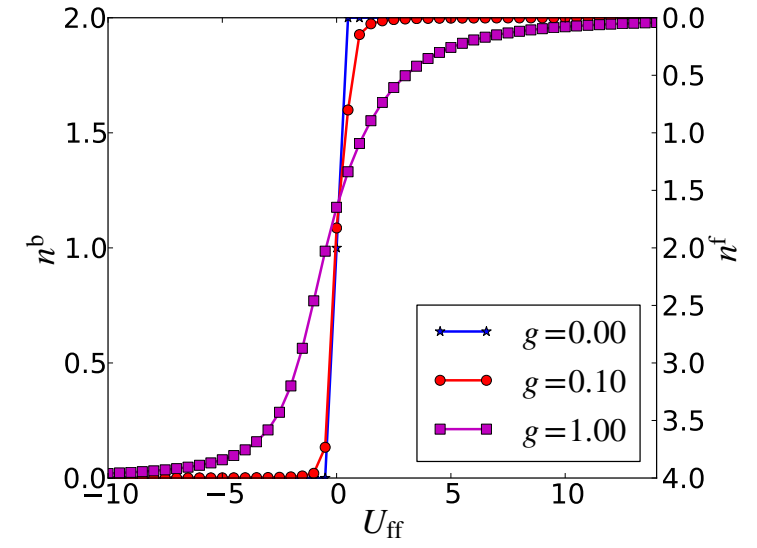

Fig. 6. The same as in Fig. 4, but for $2 N^{\mathrm{b}}+N^{\mathrm{f}}=4$ and $U_{\mathrm{bf}}=7$.

Now, the critical value of the inter-fermion interaction, at which the jump in the occupation numbers occurs for $g=0$, is $U_{\mathrm{ff}}=0$. In the limiting cases of band insulators the fermion-fermion interaction is the only factor that determines the nature of the ground state. On the one hand, if $U_{\mathrm{ff}}<0$ the energy is minimized by the presence of a maximum allowed number of fermions. On the other hand, the energy increases with the number of fermions for $U_{\mathrm{ff}}>0$ and in this case there are only bosons in the ground state. It is also possible that the kinetic energy of the intermediate state with $N^{\mathrm{f}}=2$ and $N^{\mathrm{b}}=1$ may modify this picture, but the assumed relatively strong boson-fermion repulsion $\left(U_{\mathrm{bf}}=7\right)$ makes all the particles localized in this state. Similarly to the case presented in Fig. 4, for a finite $g$ the conversion of fermions into bosons is a smoother function of $U_{\mathrm{ff}}$ than for $g=0$.

It is also possible to induce the fermion-boson conversion by controlling the inter-boson interaction $U_{\mathrm{bb}}$. This interaction, however, can be introduced only for a system with soft-core bosons. Then, the last case we study is $2 N^{\mathrm{b}}+N^{\mathrm{f}}=4$, but without the single-occupancy

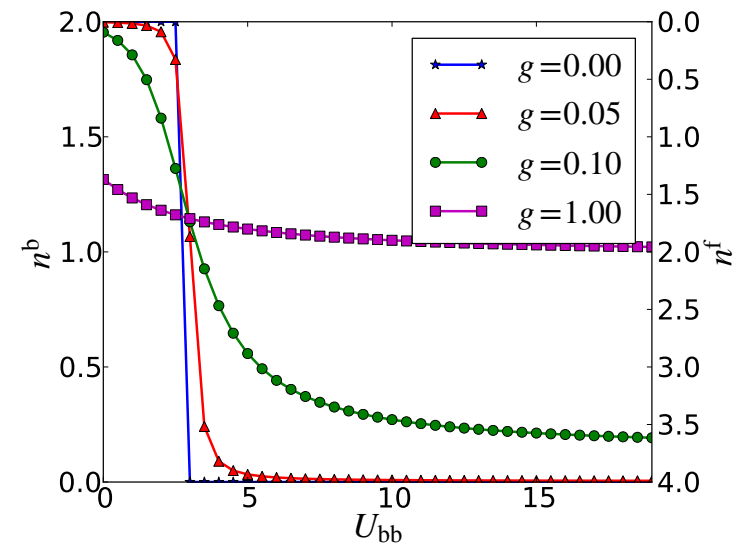

Fig. 7. The boson and fermion occupation numbers as functions of the strength of on-site interaction between soft-core bosons $U_{\mathrm{bb}} . U_{\mathrm{ff}}=0.5$ and $U_{\mathrm{bf}}=7$ has been assumed.

constraint for the bosons. Figure 7 shows the results for $U_{\mathrm{ff}}=0.5$ and $U_{\mathrm{bf}}=7$. According to the results presented in Fig. 6, for $U_{\mathrm{ff}}>0$ in the absence of the inter-boson interaction (i.e., for $U_{\mathrm{bb}}=0$ ) the ground state has a purely bosonic nature. Then, with increasing $U_{\mathrm{bb}}$ the bosons are converted into fermions. In analogy to the case of hard-core bosons, this process is abrupt for $g=0$ and smooth for $g \neq 0$. For a small $g$ the number of bosons tends to 0 for a large $U_{\mathrm{bb}}$. However, for a sufficiently strong boson-fermion coupling $g$, there is one boson and two opposite-spin fermions in the ground state in the large- $U_{\mathrm{bb}}$ limit.

\section{Summary and outlook}

In conclusion, we have analyzed a two-component model where fermions and bosons interact through both on-site Coulomb-type interactions (boson-boson, bosonfermion and fermion-fermion) and through a mechanism 
by which bosons decay in two fermions with opposite spins and vice versa. The model, studied on a small, two-site lattice, has been solved by exact diagonalization of the Hamiltonian. We have demonstrated that this model, even in the absence of the boson-fermion exchange $(g=0)$, may lead to Schrödinger-cat-like states. For $g \neq 0$ we have shown how the process of the conversion of fermions into bosons and vice versa depends on the model parameters.

While the present results cannot be directly applied to real systems in a double-well optical potential, they can be easily generalized to a larger, experimentally accessible, number of bosons. Another extension, interesting from experimental point of view, would be to take into account the inter-dimer coupling. For a few coupled dimers, exactly the same exact diagonalization approach can be applied. In a case of a larger number of weakly coupled dimers one can use the present method to find the exact eigenstates of a single dimer and then use the cluster perturbation theory to study an array of coupled dimers [24, 25].

\section{Acknowledgments}

A.B. acknowledges support by the Foundation for Polish Science under the "TEAM" program for the years 2011-2014. M.M.M. acknowledges support by the Polish National Science Center (NCN) under grant DEC2013/11/B/ST3/00824.

\section{References}

[1] M. Rinck, C. Bruder, Phys. Rev. A 83, 023608 (2011).

[2] R. Micnas, J. Ranninger, S. Robaszkiewicz, Rev. Mod. Phys. 62, 113 (1990).

[3] J. Sebby-Strabley, M. Anderlini, P.S. Jessen, J.V. Porto, Phys. Rev. A 73, 033605 (2006).

[4] M. Anderlini, P.J. Lee, B.L. Brown, J. SebbyStrabley, W.D. Phillips, J.V. Porto, Nature 448, 452 (2007).

[5] P. Cheinet, S. Trotzky, M. Feld, U. Schnorrberger, M. Moreno-Cardoner, S. Fölling, I. Bloch, Phys. Rev. Lett. 101, 090404 (2008).

[6] I. Danshita, J.E. Williams, C.A.R. Sá de Melo, C.W. Clark, Phys. Rev. A 76, 043606 (2007).

[7] S. Folling, S. Trotzky, P. Cheinet, M. Feld, R. Saers, A. Widera, T. Muller, I. Bloch, Nature 448, 1029 (2007).
[8] J. Sebby-Strabley, B.L. Brown, M. Anderlini, P.J. Lee, W.D. Phillips, J.V. Porto, P.R. Johnson, Phys. Rev. Lett. 98, 200405 (2007).

[9] S. Trotzky, P. Cheinet, S. Fölling, M. Feld, U. Schnorrberger, A. M. Rey, A. Polkovnikov, E.A. Demler, M.D. Lukin, I. Bloch, Science 319, 295 (2008).

[10] A. Avella, F. Mancini, T. Saikawa, Eur. Phys. J. B 36, 445 (2003).

[11] A. Brooks Harris, R.V. Lange, Phys. Rev. 157, 295 (1967).

[12] J. Ranninger, U. Thibblin, Phys. Rev. B 45, 7730 (1992).

[13] C. Chin, R. Grimm, P. Julienne, E. Tiesinga, Rev. Mod. Phys. 82, 1225, (2010).

[14] T. Köhler, K. Góral, P.S. Julienne, Rev. Mod. Phys 78, 1311 (2006).

[15] J.G. Danzl, E. Haller, M. Gustavsson, M.J. Mark, R. Hart, N. Bouloufa, O. Dulieu, H. Ritsch, H.-C. Nägerl, Science 321, 1062 (2008).

[16] K.-K. Ni, S. Ospelkaus, M.H.G. de Miranda, A. Pe'er, B. Neyenhuis, J.J. Zirbel, S. Kotochigova, P.S. Julienne, D.S. Jin, J. Ye, Science 322, 231 (2008).

[17] N.V. Vitanov, M. Fleischhauer, B.W. Shore, K. Bergmann, Advances in Atomic, Molecular, and Optical Physics 46, 55 (2001).

[18] J.K. Freericks, M.M. Maśka, A. Hu, T.M. Hanna, C.J. Williams, P.S. Julienne, R. Lemański, Phys. Rev. A 81, 011605 (2010).

[19] A. Hu, J.K. Freericks, M.M. Maśka, C.J. Williams, Phys. Rev. A 83, 043617 (2011).

[20] T.-L. Ho, C.V. Ciobanu, J. Low Temp. Phys. 135, 257 (2004).

[21] J. Ruostekoski, in: Directions in Quantum Optics, Eds. H.J. Carmichael, R.J. Glauber, M.O. Scully, Vol. 561 of Lecture Notes in Physics, Springer, Berlin 2001, p. 77.

[22] M.M. Maśka, R. Lemański, J.K. Freericks, C.J. Williams, Phys. Rev. Lett. 101, 060404 (2008).

[23] M.M. Maśka, K. Czajka, Phys. Rev. B 74, 035109 (2006).

[24] M.M. Maśka, Phys. Rev. B 57, 8755 (1998).

[25] D. Sénéchal, D. Perez, D. Plouffe, Phys. Rev. B 66, 075129 (2002). 